\title{
Mark A. On the Triple Square and Reynolds the Diagonal of the Golden Section
}

Geometer Mark Reynolds demonstrates the use of an ancient system for new ways of thinking and seeing: on the triple square and the diagonal of the Golden Section.

It is better to have nothing than to possess much and impart it to no one. Sextus the Pythagorean

\section{Introduction}

Last issue's focus on the Golden Section has stayed with me, and I recall doing a construction regarding a particular "action taken" on the ratio, $1.618 \ldots: 1$. For example, an action can be "taken" ${ }^{1}$ on the golden section rectangle by rotating its long side to its opposite long side to cut off the $\sqrt{ }_{\phi}$ on that opposite side. This generates both the Triangle of Price ${ }^{2}$ and the $\sqrt{ }_{\phi}$ rectangle, which is much different from the original golden section rectangle that yielded it. Only a couple of elements are brought over to the new grid. One primary similarity is the making of the golden section ratio (the "golden cut") on both rectangles as a result of a key element in the grid. In the $\phi$ rectangle, the side of the square cuts the long side of the rectangle at the golden section. In the $\sqrt{ } \phi$ rectangle, the occult centers are at the golden sections of all four sides. Most obvious is the fact that both rectangles have the same short side length of 1 .

Another action that can be taken with the rectangle is to use its diameter length (here, in the $\phi$ rectangle, the length is $1.902 \ldots)$ to become the new length of the long side of the new rectangle; its ratio then being, $1.902 \ldots$ to $1(1.902 \ldots .1$ is the ratio for the American flag!). The new system generated in the "1.902..." rectangle is the "Triple Square", a system that "grew" from the rotational action taken on the diagonal. (A similar rotational action taken on diagonals of rectangles is the Square Root Rectangle system.) In essence, the creative action of the geometer's procedures transforms (now we say "morphs") one geometric system into a totally new one. It is totally unexpected because there is no obvious external "formula" that would appear to link the two. It was through the process of drawing and construction that the metamorphosis took place.

Before beginning drawing, let's develop various portions of the rectangle's anatomy:

- We will generate the triple square from the golden section rectangle and its diagonal through the rectangle's transformation into the " $1.902 \ldots: 1$ " ratio;

- We will be separating the rectangle into its two component parts: the square and the (reciprocal) golden section rectangle;

- The diagonals and reciprocal systems become key elements in the transformation.

Here then, we proceed to the steps for our construction: 


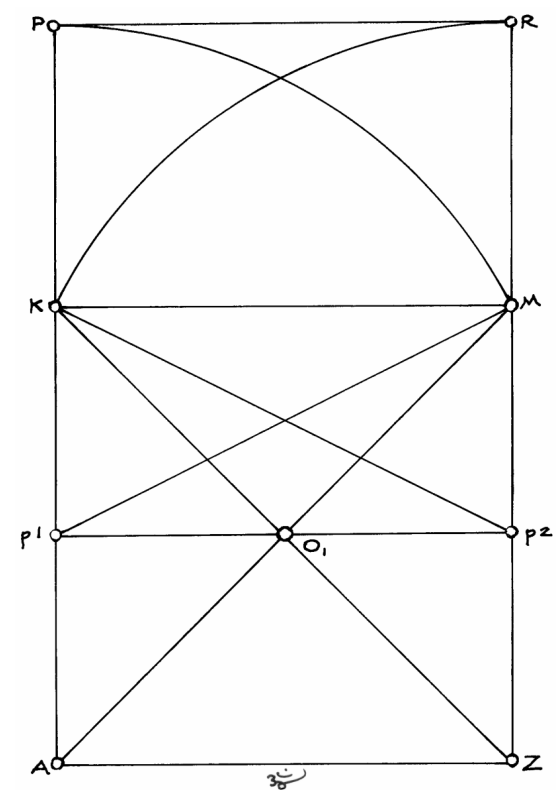

As in Fig. 1, above, construct the golden section rectangle. Here, the half-diagonals $\mathrm{p}_{1} \mathrm{M}$ and $\mathrm{p}_{2} \mathrm{~K}$ are used from the master square to generate points $\mathrm{P}$ and $\mathrm{R}$.
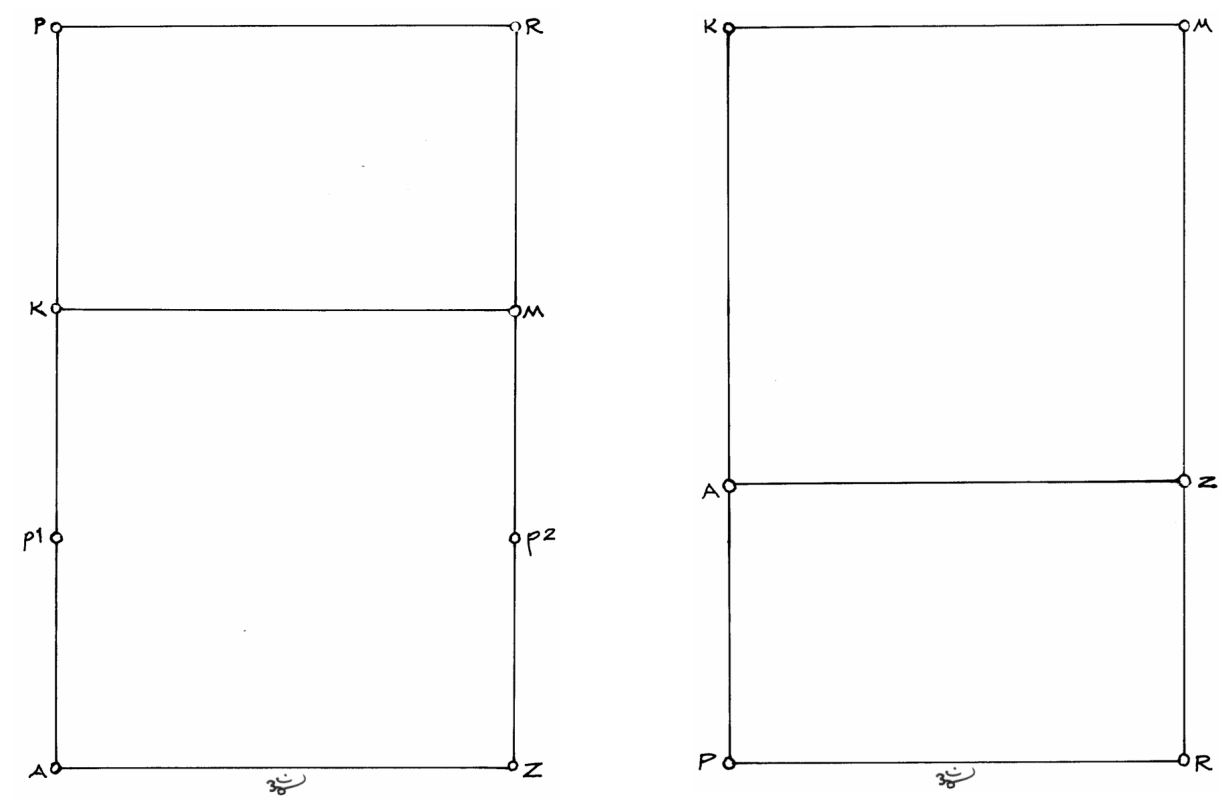

Fig. 2 (above left) and Fig. 2a (right) demonstrate the square/rectangle combination with the reversal of the positions of the square that is necessary for the transformation. 

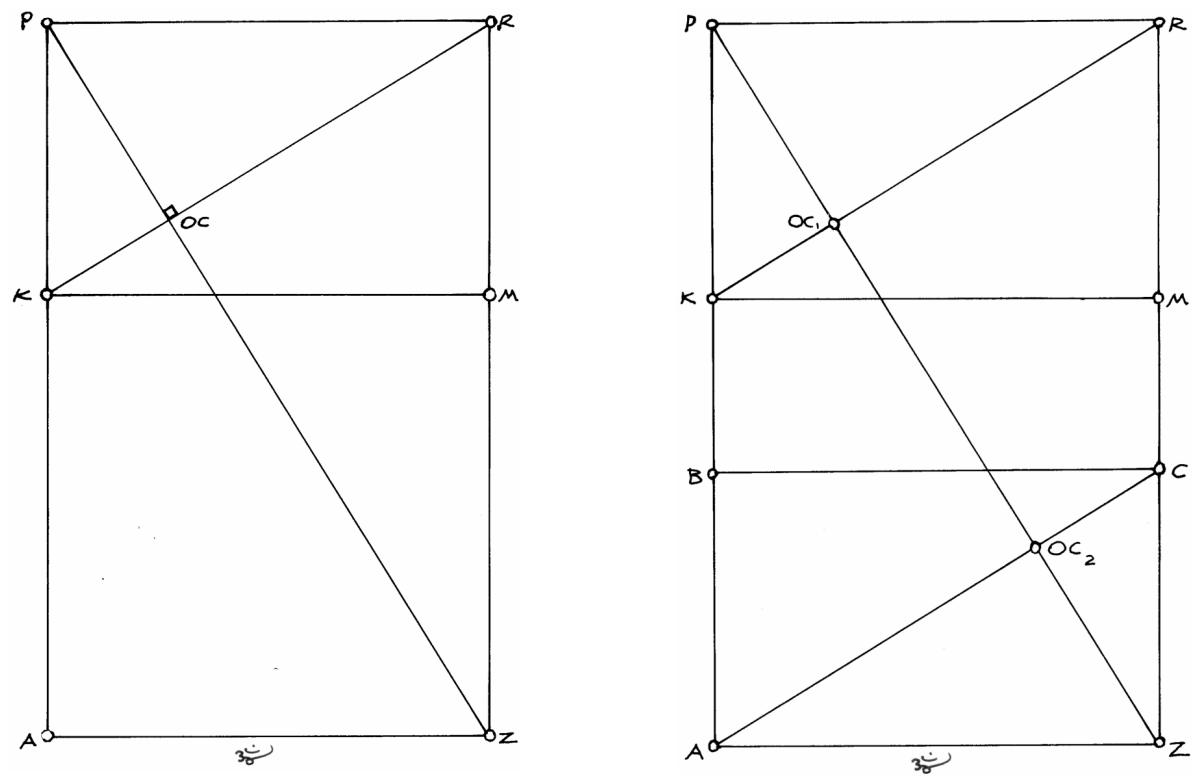

Fig. 3 (above, left) is the diagonal $\mathrm{PZ}$ and the reciprocal KR relationship. Here, the reciprocal rectangle is the golden section reciprocal rectangle that sits atop the master square, AKMZ. OC is sometimes called an "occult center." Fig. 4 (above right) introduces a second occult center, $\mathrm{OC}_{2}$.
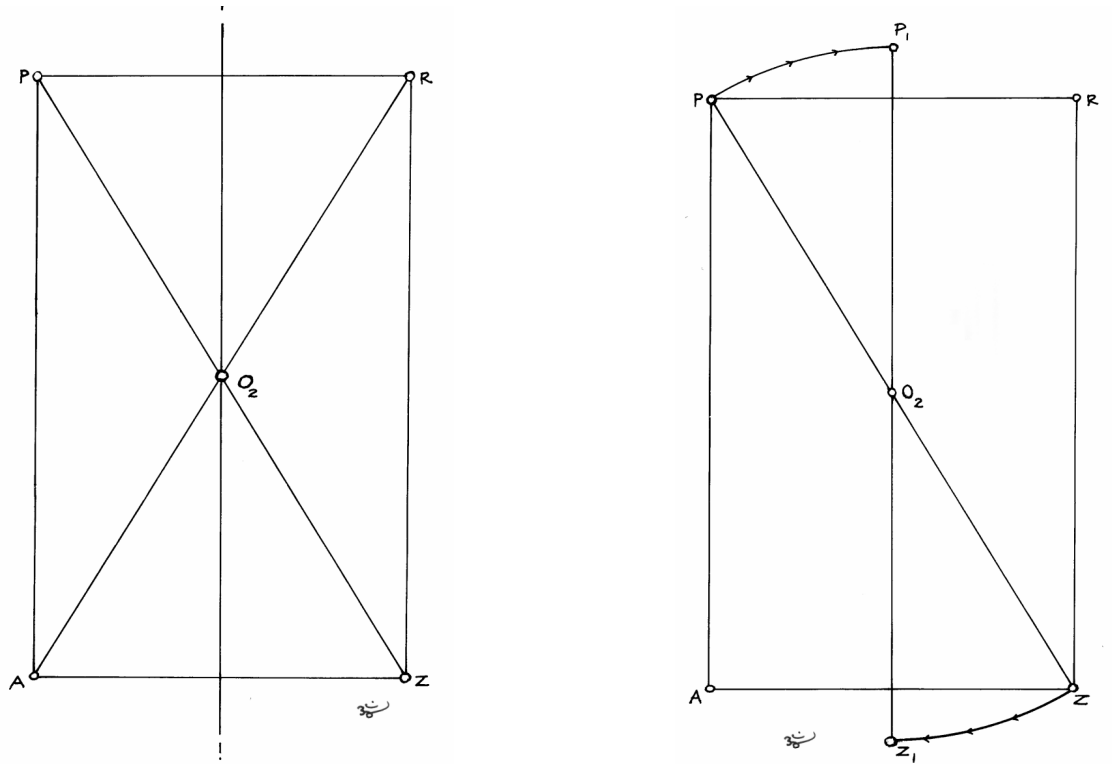

In Fig. 5 (above left), emphasis and focus now turns to rectangle APRZ. We will need the rectangle's natural center, $\mathrm{O}_{2}$. The vertical midline $\mathrm{NG}$ is also necessary for the construction. In Fig. 6 (above right) is the actual translation/rotation. With the pin of the compass in the center $\mathrm{O}_{2}$, open to the corners $\mathrm{P}$ and $\mathrm{Z}$. 

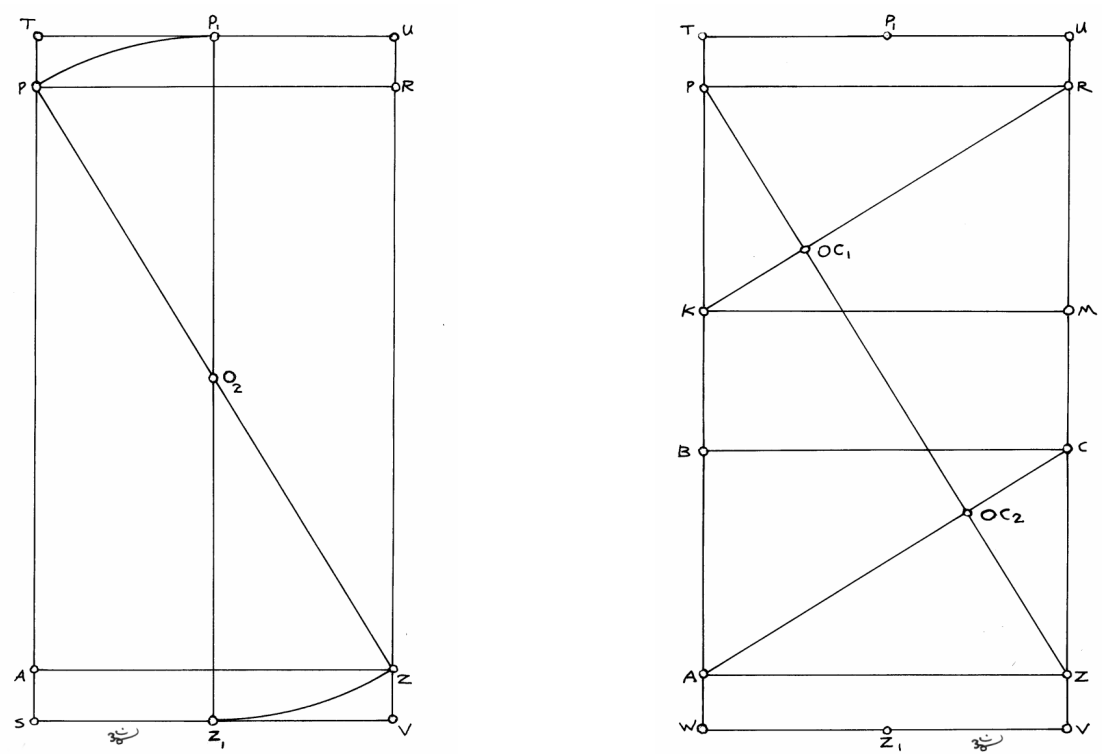

As in Figure 7 (above left), rotate line $\mathrm{PZ}$ to the vertical axis at $\mathrm{P}_{1} \mathrm{Z}_{1}$. This takes the length of the diagonal of the golden section rectangle $\mathrm{PZ}$ and length $1.902 \ldots$ and makes it the height of the new rectangle STUV. The vertical midline of the new $1.902 \ldots$ rectangle STUV is $Z_{1} P_{1}$. Fig. 8 (above right) is the construction of the $1.902 \ldots$ rectangle, with the original golden section rectangle APRZ and with the diagonal/reciprocals in the grid. (It should be noted that the two occult centers, $\mathrm{OC}_{1}$ and $\mathrm{OC}_{2}$, will play an integral part in the transformation into the triple square, and should be noted in the drawing.)

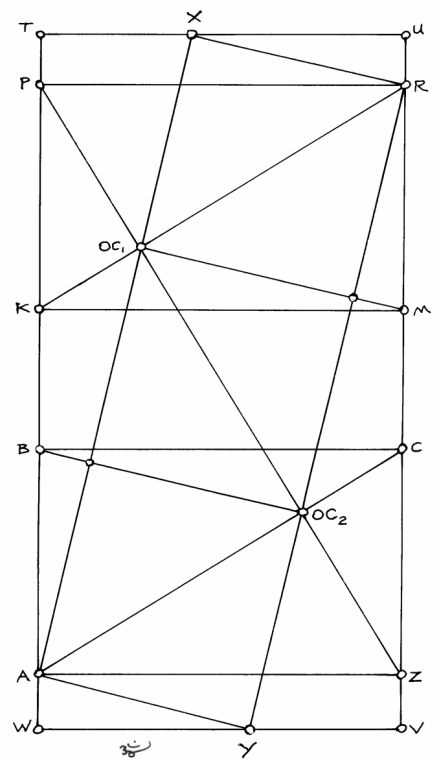

Figure 9 (left) is the actual transformation construction. Now it will be seen how the occult centers operate in the transformation. For it is from these two occult centers to the points $\mathrm{M}$ and $\mathrm{B}$ that begin the construction of the triple square. Next, from the corners of the golden section rectangle $A$ and $\mathrm{R}$, draw through the occult centers $\mathrm{OC}_{1}$ and $\mathrm{OC}_{2}$ through to the lines $\mathrm{WV}$ and $\mathrm{TU}$, intersecting them at points $\mathrm{Y}$ and $\mathrm{X}$, which form the opposite corners of the new triple square, AXRY. By lines $\mathrm{EOC}_{2}$ and $\mathrm{OC}_{1} \mathrm{M}$ divide the triple square, AXRY, into thirds-each of the three individual squares. 

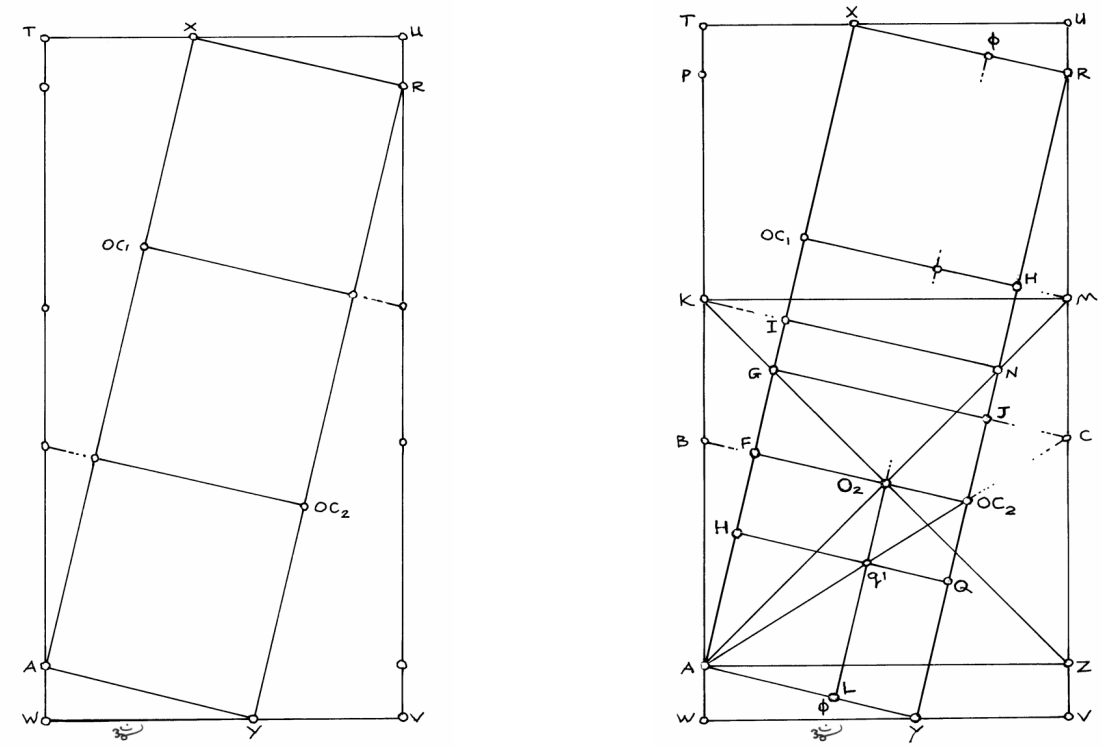

Figure 10 (above left) shows the constructional grid system for the position of the triple square (it should be noted that the triple square is dynamically positioned, that is, not vertical and horizontal) within the static (at rest) $1.902 \ldots$.rectangle. Figure 11 (above right) is but one master grid, one example, of a myriad of grids that can now be developed from the transformation.

\section{Conclusion}

Here we have a demonstration of using ancient systems for new ways of thinking and seeing. Part of my own pleasure was in finding that through all the irrational numbers and creative energy being put into the system, it generated the triple square, an elegant and beautiful rational system. It is also a good demonstration of how geometry builds things. Through the interplay of the static and dynamic elements of the grid, and the creative actions taken to rearrange and recombine them, the possibilities seem limitless. The eternal question for me remains: What is the force that initiates and enables geometry to build in such elegant and endless ways in nature?

\section{Notes}

1. There can be infinite actions taken on any and all geometric constructions, ratios, and shapes that can and will transfer them from one geometric system to another; this is to be discussed in a future column.

2. A name given to the only right triangle whose three sides are in a geometric progression: 1, $\sqrt{ }_{\phi}, \sqrt{ }^{2}$. See also my article "A Comparative Geometric Analysis of the Heights and Bases of the Great Pyramid of Khufu and the Pyramid of the Sun at Teotihuacan" in the NNJ vol. 1, no. 4 (October 1999), pp. 23-42. 


\section{About the Geometer}

Marcus the Marinite (aka Mark A. Reynolds) is a visual artist who works primarily in drawing, printmaking and mixed media. He received his Bachelor's and Master's Degrees in Art and Art Education at Towson University in Maryland. He was also awarded the Andelot Fellowship to do post-graduate work in drawing and printmaking at the University of Delaware. He is also an educator who teaches sacred geometry, linear perspective, drawing, and printmaking to both graduate and undergraduate students at the Academy of Art College in San Francisco, California. He was voted Outstanding Educator of the Year by the students in 1992. Additionally, he is a geometer, and his specialties in this field include doing geometric analyses of architecture, paintings, and design. For the past decade, Mr. Reynolds has been at work on an extensive body of drawings, paintings and prints that incorporate and explore the ancient science of sacred, or contemplative, geometry. He is widely exhibited, showing his work in group competitions and one person shows, especially in California. His work is in corporate, public, and private collections, and he is represented by the Mill \& Short Gallery in San Francisco. He is a member of the California Society of Printmakers, the Los Angeles Printmaking Society, and the Marin Arts Council. He published "A Comparative Geometric Analysis of the Heights and Bases of the Great Pyramid of Khufu and the Pyramid of the Sun at Teotihuacan" in the NNJvol.1 (1999). 